\title{
Gravitational Waves: Present Status and Future Prospectus
}

\author{
Naseer Iqbal*, Showkat Monga \\ Department of Physics, University of Kashmir, Srinagar, India \\ Email: ${ }^{*}$ dni phtr@kashmiruniversity.ac.in \\ Received $* * * * 2014$ \\ Copyright (C) 2014 by authors and Scientific Research Publishing Inc. \\ This work is licensed under the Creative Commons Attribution International License (CC BY). \\ http://creativecommons.org/licenses/by/4.0/

cC) (i) Open Access

\begin{abstract}
Remarkable progress has been made during recent years on the development of gravitational wave detectors. The review describes the present status and future prospectus of the gravitational wave astronomy. The main theme is to review the prominent long baseline detectors in operation around the world and proposed baseline and space-borne interferometers. Looking to the future, the major upgrades to the ground based detectors and new planned detectors will be completed over the coming years, which will create a network of detectors with the significantly improved sensitivity required to detect gravitational waves. Beyond this, the concept and design of possible future "third generation" gravitational-wave detectors will be discussed. Sources such as coalescing compact binary systems, neutron stars in low-mass X-ray binaries, stellar collapses and pulsars are all possible candidates for detection.
\end{abstract}

\section{Keywords}

Gravitational Waves; General Relativity; Detectors; Electromagnetic Waves

\section{Introduction}

Gravitational radiation is a central and exotic prediction of general theory relativity proposed by Einstein and its detection is a key test of integrity of his work. Study of gravitational waves is presently considered as one of the major challenges in theoretical physics and efforts are made to gather and analyze all kinds of related information about them. The first paper about the idea of gravitational waves was published by Laplace in 1776. After many attempts to develop a relativistic theory of gravity by various authors, Einstein proposed general theory of relativity. Soon after his proposal of theory, Einstein predicted that existence of gravitational waves and estimated its strength.

${ }^{*}$ Corresponding author. 
Sources like interacting black holes, coalescing compact binary systems, stellar collapses and pulsars are all possible candidates for detection. The signals received from them will significantly increase our understanding of the universe. General theory of relativity predicts gravitational waves as freely propagating "ripples" in space time and manifests themselves as fluctuating tidal forces on masses in the path of the wave like other waves. Their detection is primarily an experimental science, consisting of the development of the necessary ultra-sensitive measurement techniques. While the gravitational waves can be considered as classical waves, the measurement systems must be treated quantum mechanical.

Gravitational waves which travel with speed of light are quite different from electromagnetic waves. Electromagnetic waves originate from excited atoms and molecules, whereas gravitational waves are emitted by acelerated massive objects. Also electromagnetic waves are easily scattered and absorbed by dust clouds between the objects and the observer, whereas gravitational waves will pass through them almost unaffected, therefore giving rise to the expectation that the detection of gravitational waves will reveal a new and different view of the universe. The only experimental evidence for gravitational waves comes from timing of binary pulsar system [1] [2]. These systems consist of two neutron stars orbiting each other. To be observable one of them must be active and emit radio waves. The first double pulsar system PSR B $1913+16$ was discovered by Hulse and Taylor in 1974 [3]-[5]. It is located in the milky way, its orbital period is $\sim 8$ hours and the received radio signal repeats itself at a rate of $\sim 17 / \mathrm{sec}$. The emission of gravitational waves brings the two neutron stars closer together and this increases the orbital frequency. Observations of the orbit of the binary pulsar over the succeeding years have shown that the orbital period is decreasing at a fractional rate $(2.71 \pm 0.10) \times 10^{-9}$ per year (as shown in Figure 1). General relativity predicts an orbital decay rate due to gravitational wave emission of $(2.715 \pm 0.002) \times 10^{-9}$ per year. This agreement is a most impressive and beautiful conformation of the theory and provides strong evidence for the existence of gravitational waves. The loss of potential energy in this system is in agreement with the emission of gravitational waves predicted by general relativity theory [2] [6]-[8]. As a consequence the two neutron stars will merge in about 300 million years. The only difficulty is that most of the systems like the Hulse-Taylor binary are far away. The amplitude of waves given off by Hulse-Taylor binary as seen on earth would be roughly $h \sim 10^{-26}$.

There are some sources, however, that astrophysicists expect to find with much larger amplitude of $h \sim 10^{-20}$.

Indeed it is not unreasonable to expect that gravitational waves will become a powerful tool for astronomy, revealing features of their sources which one could never learn by electromagnetic waves [9], cosmic ray, or

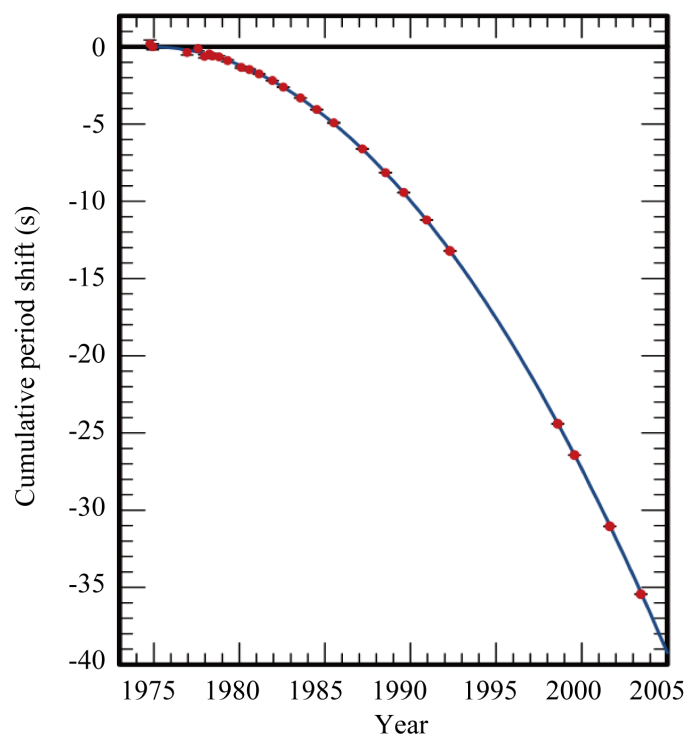

Figure 1. Orbital decay of PSR $1913+16$ binary pulsar systems, from Data points represent the cumulative shift of periastron time measured whereas the parabola curve shows the same quantity predicted by the General Relativity. Courtesy: LIGO Hanford Observatory Richland, WA 99352, USA. 
neutrino studies.

The existence of gravitational waves predicted by Einstein in 1916 has not been confirmed directly even after 100 years of his prediction, but an indirect measurement of gravitational waves has been performed. To observe and to detect gravitational waves in the near future from various different astrophysical and cosmological sources in different ranges and to enhance the signal to noise ratio's, we need to explore the gravitational wave spectrum between the high frequency band and the very low frequency band. The usual way of detection of gravitational wave is by measuring the strain $\Delta l / l$ induced by it. Hence gravitational wave detectors are usually amplitude sensors, not energy sensors.

First attempts of detecting gravitational wave have been done in 1960's with the help of resonant bars but they have been unable to provide a direct detection of gravitational radiation [10]. During the 1960's, the idea of detecting the space time ripples induced by the gravitational radiations with laser interferometry aroused [11]. It was followed by the construction of a network of laser interferometric gravitational wave detectors in the 2000 s. On the time scale of a few years, several ground based detectors were put online, out of whom CLIO, GEO 600, VIRGO, MiniGRAIL are operational, whereas initial and enhanced LIGO \& TAMA 300 have been decommissioned. However mission concepts under implementation are LCGT/KAGRA, advanced LIGO [12]. Advanced VIRGO, GEO-HF, AIGO, ET, INDIGO. Nevertheless, with ground based detectors it seems impossible to go below the $10 \mathrm{~Hz}$ limit due to gravity gradient noise as well as technological limits. The best solution is to fly a laser interferometer in space. Mission concepts under consideration and study are LISA, ASTROD, [13] SuperASTROD [14] [15], ASTROD-GW [16], BBO [17] and DECIGO [18].

\section{Classification of Gravitational Waves}

Similar to frequency classification of electromagnetic waves, we can have the following frequency classification of gravitational waves [19].

a) Ultra high frequency band (above $1 \mathrm{THz}$ ); detection methods include Terahertz resonators, optical resonators and ingenious methods to be invented.

b) Very high frequency band (100 KHz - $1 \mathrm{THz}$ ); Micro wave resonators/waves guide detectors, optical interferometers and Gaussian beam detectors are sensitive to this band. High frequency band (10 Hz - $100 \mathrm{KHz}$; low temperature resonators and laser interferometer ground detectors are the most sensitive to this band.

c) Middle frequency band $(0.1 \mathrm{~Hz}-10 \mathrm{~Hz})$; space interferometry detectors of short arm length (1000 - 100000 $\mathrm{km})$ are most sensitive to this band.

d) Low frequency band $(100 \mathrm{nHz}-0.1 \mathrm{~Hz})$; laser interferometer space detectors are most sensitive to this band.

e) Very low frequency band (300 $\mathrm{PHz}-100 \mathrm{nHz}$ ); pulsar timing observations are most sensitive to this band.

f) Ultra low frequency band (10 fHz - $300 \mathrm{PHz})$; astrometry of quasar proper motion is most sensitive to this band.

g) Extremely low (Hubble) frequency (1 aHz - $10 \mathrm{fHz}$ ); cosmic micro wave background experiments are most sensitive to this band.

h) Infra Hubble frequency band (below $1 \mathrm{aHz}$ ); inflationary cosmological models give strengths of gravitational waves in this band. They may be verified indirectly through the verification of inflationary cosmological model [20].

This paper does not attempt to coverall or even much of the primary literature. In essence, this paper is a review of present status and future prospectus of gravitational waves.

\section{Gravitational Waves and General Relativity}

General theory of relativity, published by Albert Einstein in 1915, is a classical field theory of gravitation. It is beyond Newton's theory, which is valid only for particles moving with slow velocity in a weak and static gravitational field. When it comes to situations involving strong gravity, such as compact stellar objects and cosmology, or time dependent gravitational fields as in emission and propagation of gravitational waves, the use of general relativity is indispensable.

Einstein in general relativity describes the space-time geometry as being determined by the energy matter distribution of a system of massive body. The curvature of the space-time metric is linked to the energy content of the universe by the equation 


$$
G_{\mu v}=R_{\mu v}-\frac{1}{2} g_{\mu v} R=-\frac{8 \pi G}{C^{4}} T_{\mu v}
$$

where $G_{\mu \nu}$ is the Einstein tensor describing space-time geometry as the sum of the Ricci tensor $R_{\mu \nu}$ and of the metric tensor $g_{\mu v}, G$ is the gravitation constant and $T_{\mu \nu}$ is the stress energy tensor. It represents the source of the gravitational waves.

The metric tensor $g_{\mu v}$ representing the geometry of space-time connects space-time co-ordinate $d x^{\mu} \quad(\mu, v=$ $1,2,3,4)$ to the space interval " $d s$ ” by way of the relation.

$$
d s^{2}=g_{\mu v} d x^{\mu} d x^{v}
$$

It being real that the gravitational waves in the vicinity of the earth will always be very weak, therefore the background curvature can be ignored and the background metric can be approximated as that of Minkowski flat metric $\eta$.

The Einstein equation can be linearized in the case of small perturbation. The gravitational wave field by an approximation can then be expressed as

$$
g_{\mu v}=\eta_{\mu v}+h_{\mu v}
$$

where $\eta_{\mu v}$ the metric of the flat background and $h_{\mu v} \ll 1$ is the small perturbation induced by the gravitational waves. To obtain an explicit statement of the metric perturbation $h$ it is necessary to make a gauge choice. The most useful gauge is the transverse traceless gauge in which the coordinates are defined by the geodesis of freely falling test bodies. In this gauge, and in the weak field limit, the solution of Equation (1) wherein the equation of general relativity become a system of linear equations, specifically a system of wave equations.

$$
\left(\nabla^{2}-\frac{1}{c^{2}} \frac{\partial^{2}}{\partial t^{2}}\right) h_{\mu v}=0
$$

It represents three dimensional wave equation indicating that gravitational waves travel at the speed of light.

The general solution of Equation (4) is a superposition of monochromatic plane wave, a single monochromatic plane wave can be written as:

$$
h_{\mu v}=\left(h_{+} \epsilon_{\mu \nu}^{+}+h_{\times} \epsilon_{\mu \nu}^{\times}\right) \mathrm{e}^{-i\left(\Omega_{G W} t-k_{G W} x\right)}
$$

With $\Omega_{G W} \& K_{G W}$ being respectively the angular frequency and the wave vector of gravitational wave. $\epsilon_{\mu v}^{+} \&$ $\epsilon_{\mu v}^{\times}$are the polarization tensor of the wave supposed propagating along $z$ axis.

The wave field is transverse and traceless and for waves travelling in the $Z$ direction may be expressed as

$$
h_{\mu v}=h_{+}\left(t-\frac{z}{c}\right)+h_{\times}\left(t-\frac{z}{c}\right)=\left(\begin{array}{cccc}
0 & 0 & 0 & 0 \\
0 & h_{x x} & h_{x y} & 0 \\
0 & h_{y x} & h_{y y} & 0 \\
0 & 0 & 0 & 0
\end{array}\right) \mathrm{e}^{i(\omega t-k x)}
$$

It represents a quadrupole wave and has a particular physical interpretation (as shown in Figure 2).

The wave being transverse in nature has no $Z$ component and to the traceless " $h$ " satisfies

$$
h_{x x}=-h_{x y}
$$

Because the Riemann Tensor is Symmetric, “ $h$ ” also satisfies

$$
h_{x y}=h_{y x}
$$

The symmetry of " $h$ " means that there are just two possible independent polarization states which are usually denoted by $h_{+}$and $h_{\times}$. In this case of sinusoidal gravitational waves, we can express these polarization as:

$$
\begin{aligned}
& h_{+}=h_{x x}=\operatorname{Re}\left[A_{+} \mathrm{e}^{-i \omega(t-z / c)}\right] \\
& h_{x}=h_{x y}=\operatorname{Re}\left[A_{x} \mathrm{e}^{-i \omega(t-z / c)}\right]
\end{aligned}
$$

Here $A_{+}$and $A_{\times}$are the strain amplitudes of each polarization. 


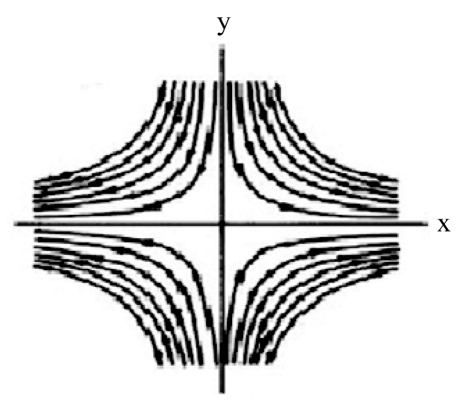

(a)

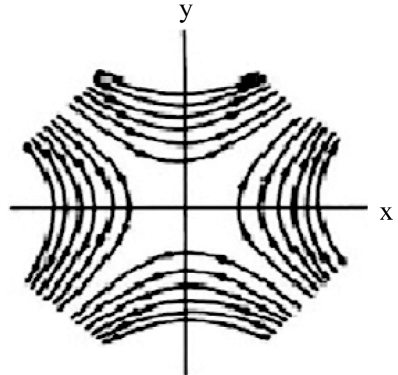

(b)

Figure 2. Direction of space deformation for a gravitational wave propagating along the $z$-axis, + polarization (a) and $\times$ Polarization (b). Courtesy: LIGO Hanford Observatory Richland, WA 99352, USA.

\section{Present Status}

Scientists have been hunting gravitational waves for years and possibly are on the verge of a break through. In the coming times, super-sensitive arrangement of ground based and space based instruments will observe the gravitational wave sky and will discover unexpected phenomenon which will provide new insight into many of the most profound astrophysical phenomena known.

During recent years a shift in the technologies used in gravitational wave searches has been observed, as the first generation gravitational wave interferometers have begun operation, taking up the baton from the bar detectors that pioneered the search for the first direct detections of gravitational waves. These ground based $\mathrm{Km}$-Scale interferometers and their advanced upgrades will be critical in establishing the field of gravitational wave astronomy.

In the US, the Laser Interferometer gravitational wave observatory (LIGO) consists of three multi kilometer scale interferometers. The Hanford, Washington, WA site has two co-located and co-aligned interferometers, one with $4 \mathrm{~km}$ long arms and another with $2 \mathrm{~km}$ long arms, referred to as H1 and H2, respectively. The Livingston, Louisiana, LA site has one interferometer with $4 \mathrm{~km}$ arms called L1. In Europe, VIRGO is a multi Kilometer scale interferometer located near Pisa Italy and GEO600, a Kilometer scale interferometer is located near Hannover Germany. The TAMA detector, located near Tokyo Japan is half of size of GEO600.

CLIO is the cryogenic Laser Interferometer Observatory. It is testing cryogenic mirror technologies for the future large cryogenic Gravity telescope (LCGT). It is situated 1000m underground in the Kamioska mine Japan.

Mini GRAIL is the first detector to use a spherical design. It is located at Leiden University in the Netherlands.

It is hoped that the first direct observation of gravitational waves will be made in the next few years by this international network of detectors. The low frequency range below about $1 \mathrm{~Hz}$ includes a large and diverse population of strong gravitational waves sources that can only be observed at these frequencies. Detection technologies are diverse. All of these technologies will eventually be used to observe the complete gravitational wave spectrum covering more than 20 orders of magnitude in frequency. LISA, a space base interferometer will open the low frequency gravitational window from $0.1 \mathrm{mHz}$ to $0.1 \mathrm{~Hz}$. The Scientific community has prioritized some future planes for successful observations of gravitational waves.

The main limitation to the sensitivities of current interferometric detectors is seismic noise, thermal noise in the mirrors and suspensions and photoelectron shot noise in the detecting photo diodes. A further enhancement to sensitivity may be obtained by recycling a signal sideband by a mirror placed at the output of the detector before the detection photo diode. This technique of 'Signal recycling” has been used to date only in the German/ UK GEO600 detector but is planned for incorporation in future detector upgrades.

A comprehensive review of main interferometer projects is as under:

\subsection{Ligo}

The LIGO mission is to directly observe gravitational wave of cosmic origin. The LIGO detectors located in 
USA have the Fabry-Perot Michelson configuration with power recycling. A highly stabilized $10 \mathrm{w}$ Nd: YAG Laser operating at $1.06 \mu \mathrm{m}$ strikes the beam splitter and is directed to the two arms. The LIGO detectors were designed so that the dominant noise would be seismic motion at low frequencies, thermal noise at intermediate frequencies and shot noise at the highest frequencies, to provide a sensitive band between approximately $40 \mathrm{~Hz}$ and 6 kHz. Cofound in 1992 by Kip Thorne \& Ronald Drever of Caltech and Raina Weiss of MIT started its observation in 2002 and ended in 2002; no unambiguous detection of gravitational waves have been reported.

In August 2010 a radio Pulsar was discovered through analysis of data from radio telescopes by the LIGO collaboration. The detection was made using the 'Einstein @ home” personal computer collaboration through LIGO \& BOINC. Although significant discovery of this radio Pulsar was not a detection of gravitational wave nor did it involve the LIGO interferometer.

Till date, LIGO has completed six science runs. The first five were known as initial LIGO. In fifth science run $S_{5}$ which lasted from November 2005 to September 2007 the LIGO detectors attained their design sensitivity with strain amplitude of $10^{-21}$ in the $40-7000 \mathrm{~Hz}$ frequency band [21]-[23] (As shown in Figure 3). A number of searches for gravitational waves from various sources have already been conducted with data from LIGO and its international partners [24]-[28]. Thereafter $\mathrm{S}_{6}$ also known as enhanced LIGO lasted from July 2009 to October 2010. Hardware was improved in LIGO for science run $S_{6}$. After the completion of $S_{5}$, the two LIGO detec-

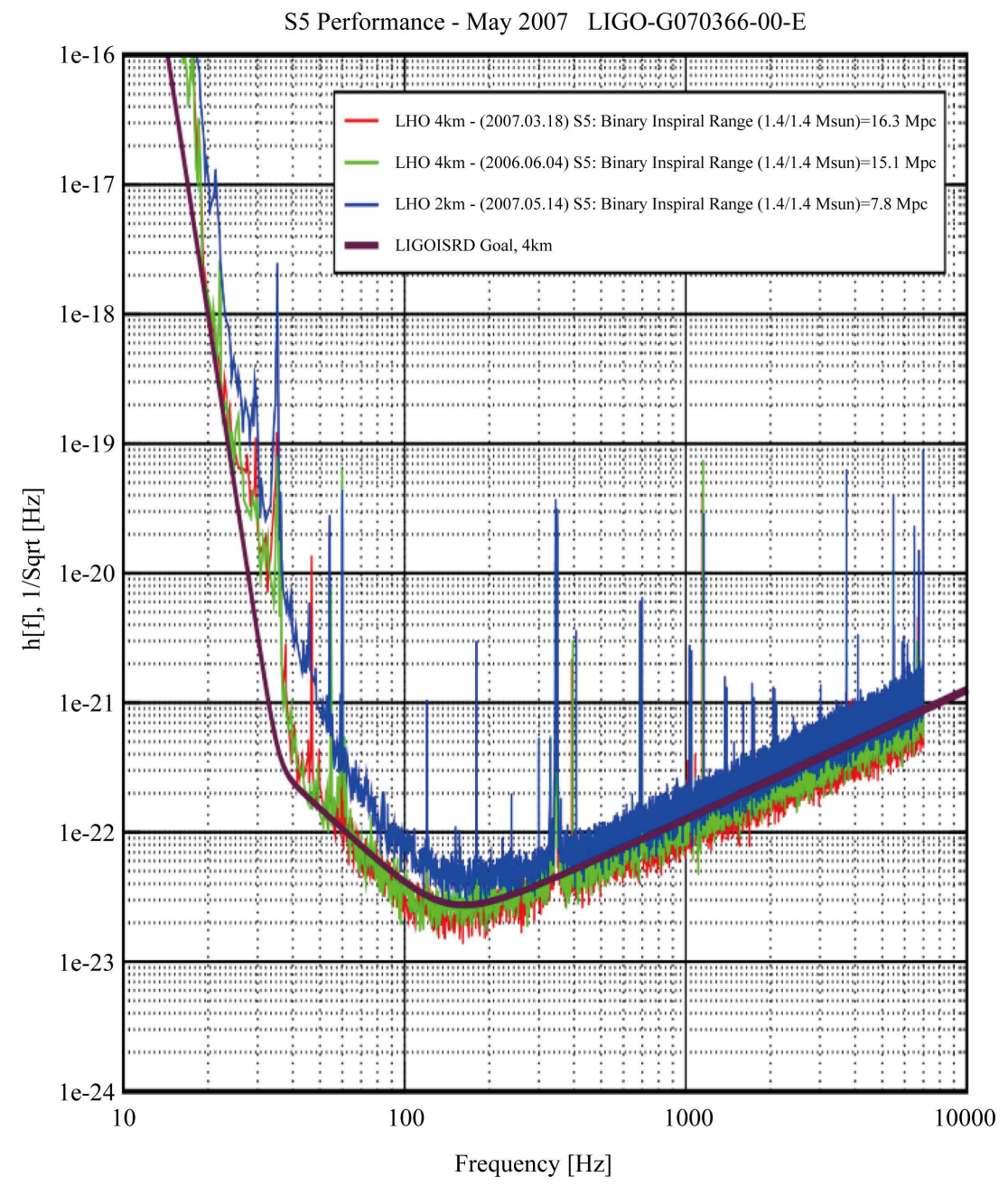

Figure 3. Measured sensitivity of the initial LIGO interferometers during S5 in strain amplitude spectral density, compared to the design sensitivity goal for an initial LIGO interferometer with $4 \mathrm{~km}$ arms. Also shown are the calculated distances to which each instrument could detect the inspiral of a $1.4 / 1.4 \mathrm{M}$ binary system of neutron stars. Courtesy: www.ligo.caltech.edu. 
tors with 4 km arms, H1 and L1, were taken offine for upgrades based on knowledge of the noise sources limiting initial LIGO, the upgrades will improve their Sensitivity by roughly a factor of 2. This program was named as Enhanced LIGO. The third detector, H2, was left on-line, in tandem with GEO600 to observe until the upgrades are complete. In parallel with these activities, construction of Advanced LIGO has begun. Advanced LIGO will use the initial LIGO buildings and vacuum systems but will otherwise consist of completely new instruments. All three interferometers will be upgraded and the arms of $\mathrm{H} 2$ will be extended to $4 \mathrm{~km}$.

The proposed LIGO-India project aims to move one advanced LIGO detector from Hanford to India. LIGO-India project is envisaged as an international collaboration between LIGO Laboratory and three lead institutions in the Indigo consortium. The motivation behind this project is to expand the world wide activities in gravitational wave detection to produce an effective global network.

\subsection{Virgo}

Virgo is a Michelson based interferometer with $3 \mathrm{Km}$ long arms located near Pisa-Italy. Construction of Virgo, approved by CNRA and INFN in 1993 started at the Cascina site in 1996.Virgo is managed by the European Gravitational observatory (EGO). The construction completed in 2003 June was followed by a commissioning phase to optimize the control systems of the detectors. Several major upgrades brought Virgo to its final configuration by the end of 2005 [29]-[31].

Virgo has completed three science runs, Virgo first science run (VSRI) was in coincidence with $\mathrm{S}_{5}$ which lasted from May 2007 to October 2007 [32] [33]. During this period, joint LIGO Virgo searches for gravitational waves were carried out. Virgo second and third science runs (VSR2) \& (VSR3) were carried from July 2009 to January 2010 and from August 2011 to October 2011 respectively. During these runs the Virgo detector operated with more sensitivity than VSR1. During VSR1, the science-mode duty factor was 81\% and by the end of the run maximum Neutron-star-binary inspiral range was frequently up to about $4.5 \mathrm{Mpc}$. The best sensitivity curves

for WSR1, WSR10 and VSR1 can be seen in Figure 4.

LIGO has completed science run $\mathrm{S}_{6}$ is now currently preparing for the advanced LIGO era, which possibly may begin by 2014. Virgo will also be upgraded on the same schedule as advanced LIGO [33] [34]. This will start the era of joint analysis between LIGO and Virgo detectors. The first upgrade Virgo ${ }^{+}$was installed during summer 2008. Thanks to joint LIGO \& Virgo data, an upper limit has been set to the gravitational wave emission of the vela [34] and Crab. The detector is currently running day and night listening for any and all gravitational signals which may arrive at any time from any part of the Universe. The signals are detected and recorded and pre analyzed through an online computing system for further analysis.

\subsection{TAMA}

The TAMA project was initiated in 1995. The baseline length is 300m and is placed underground at the Mitaka Campus of the National Astronomical Observatory of Japan (NAOJ). Like LIGO and VIRGO, TAMA is power recycled Michelson Fabry-Perot system. Over the period between August 1999 to January 2004 nine data taking runs were conducted by TAMA, several of these in coincidence with LIGO [35]. After the last run, TAMA paused in its observational phase to allow a period of noise reduction efforts. The primary focus was to reduce the effect of stray light beam and install an improved system to further isolate the test mass mirrors in the detector from ground vibrations. This is in final stage of commissioning to allow improved sensitivity for TAMA.

\subsection{GEO}

GEO600 is a German/UK interferometer of $600 \mathrm{~m}$ arm length situated near Hannover. Construction and installation of GEO600 concluded in 2002 with increased Laser power, installation of monolithic suspension for the end test masses [36]. GEO uses a three bounce, four beam, and delay line in each arm and both power recycling and signal recycling are incorporated. For the science run S1, carried out in coincidence with LIGO, the detector was kept in this configuration. Following S1 the signal recycling mirror was installed and during late 2003 the first lock of the fully dual-recycled system was achieved [37] [38]. Other upgrades included the installation of the final mirrors, suspended as triple pendulums, and with monolithic final stages. After their installation it was realized that the radius of curvature of one of the mirrors mismatched, due to which GEO600 did not participate in the S2 run. Very soon after proper rectification GEO600 took part in the S3 run in two time intervals from 5 - 11 


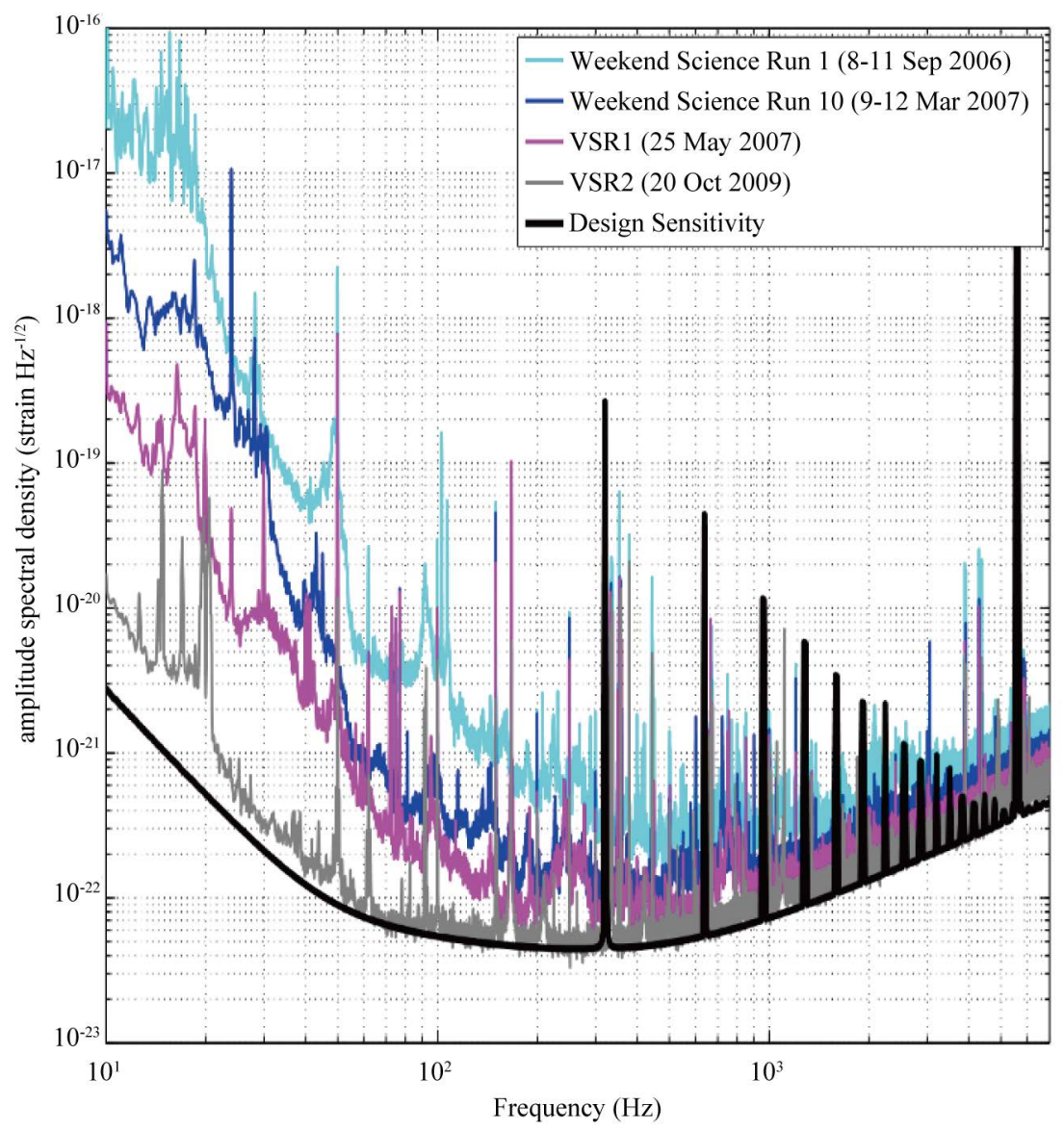

Figure 4. The best strain sensitivities from the Virgo weekend and full time science runs WSR1, WSR10, VSR1 and VSR2. Courtesy: www.geo600.org.

November 2003, dubbed S3I, and from 30 December 2003 to 13 January 2004, dubbed S3II. During S3I GEO600 operated with the signal-recycling cavity tuned to $\sim 1.3 \mathrm{kHz}$, and had a $\sim 95 \%$ duty factor, but was then takenoff-line for more commissioning work. In the period between S3I and II various sources of noise and lock loss were diagnosed and mitigated, including noise from a servo in the signal recycling cavity and electronic noise on a photo-diode. This leads to improved sensitivity by up to an order of magnitude at some frequencies (as shown in Figure 5).

Due to the upgrades, had an increased duty factor of $\sim 99 \%$. GEO600 took part in the science run $\mathrm{s}_{4}$ in coincidence with LIGO from 22 February to 24 March 2004, with a 97\% duty factor.

The main changes to the detector after S4 were to shift the resonance condition of the signal recycling cavity to lower frequency, $350 \mathrm{~Hz}$, allowing better sensitivity in the few hundred $\mathrm{Hz}$ regime, and increasing the circulating laser power, with an input power of $10 \mathrm{~W}$. GEO600 joined S5 at the later stage from 21 January 2006 to take data during nightand-weekend. For S5 the signal recycling cavity was re-tuned up to $550 \mathrm{~Hz}$. It went into full-time data taking from 1 May to 16 October 2006, with an instrumental duty factor of 94\%. The average peak sensitivity during S5 was better than $3 \times 10^{-22} \mathrm{~Hz}^{-1 / 2}$. After this it was deemed more valuable for GEO600 to continue more noise hunting and commissioning work, to give as good sensitivity as possible for when the LIGO detectors went offline for upgrading. However, it did continue operating in night-and-weekend mode.

GEO600 continued operating in Astrowatch mode between November 2007 and July 2009 after which upgrades began. This project is called GEO-HF [39]. The Upgrading towards GEO-HF has been taking place since summer 2009. The main upgrades started during 2009 were to change the read-out scheme from an RF read-out to a DC read-out system [40]. GEO-HF participated in S6 in an overnight and weekend mode, alongside a commissioning schedule, and is continuing in this mode following the end of S6. The GEO collaboration is a member of the LIGO scientific collaboration (LSC) and the data collected by the network are analyzed by the mem- 


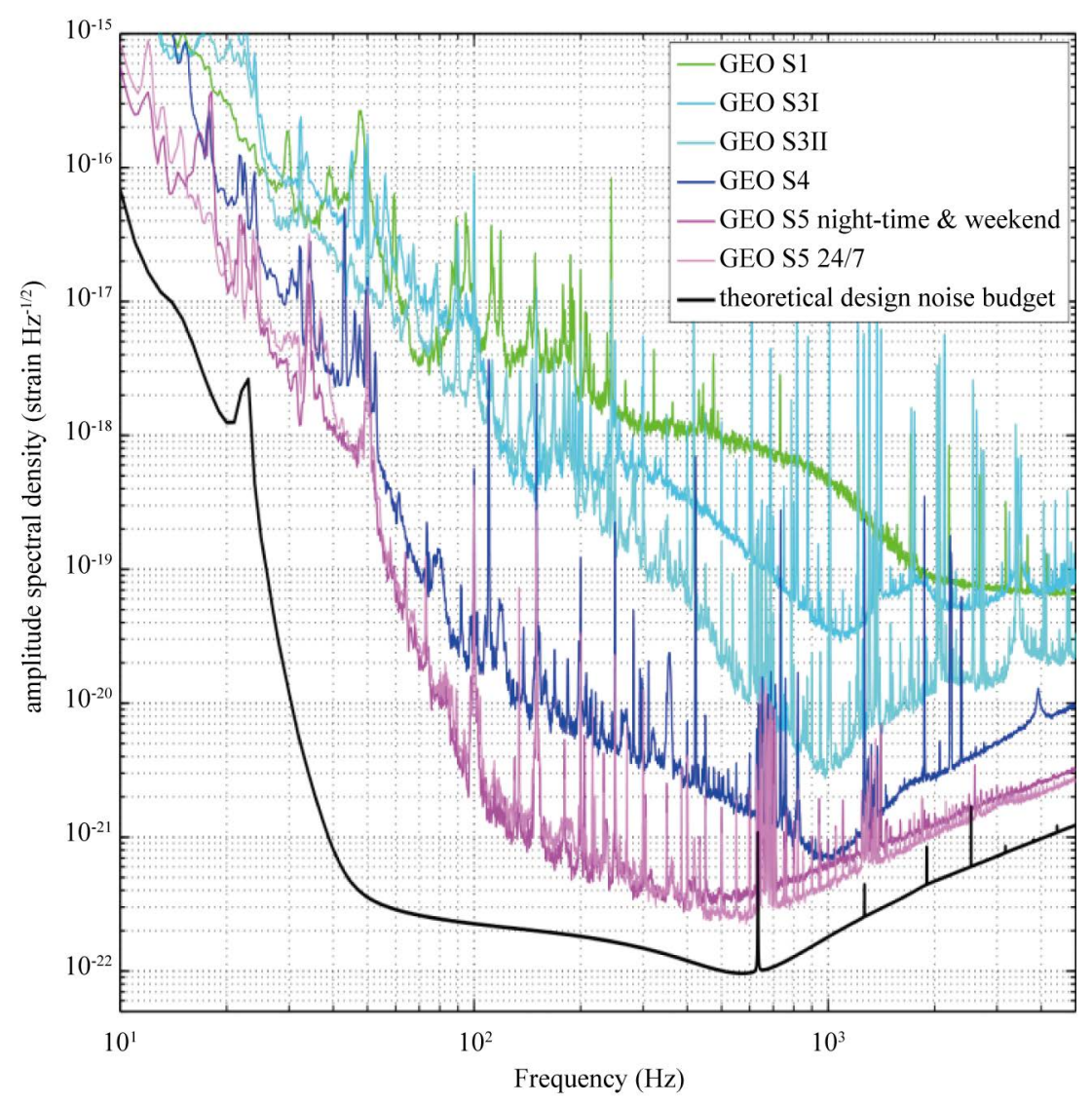

Figure 5. For S3II the signal recycling cavity was tuned to $1 \mathrm{kHz}$ and the typical strain sensitivities from the GEO600 science runs S1 through S5. Courtesy: www.geo600.org.

bers from LIGO and GEO within the LSC. In 2006 \& 2007 work at GEO 600 focused on the fifth LIGO scientific collaboration data taking run $\left(\mathrm{S}_{5}\right)$ in coincidence with the three LIGO detectors.GEO600 is operational as on date.

\subsection{CILO}

Cryogenic Laser Interferometer observatory is an optical interferometer with two perpendicular arms $100 \mathrm{~m}$ long with mirrors cooled at $20 \mathrm{k}$ situated $1000 \mathrm{~m}$ underground in the Kamioka mine [41]. This is to demonstrate the very stable conditions (i.e., low levels of seismic noise) existing in the mine and also the cryogenicallycooled sapphire mirrors suspended fromaluminum wires. In experiments with CLIO at room temperature (i.e. $300 \mathrm{~K}$ ), using a metallic glass called Bolfur for its wire suspensions, it has already been used to produce an astrophysics result by looking for gravitational waves from the Vela pulsar [42]. Tests with the cryogenic system activated and using aluminum suspensions allowed two mirrors to be cooled to $\sim 14 \mathrm{~K}$. CILO is one of the science facilities for physics at the Institute of Cosmic Ray research of the University of Tokyo.

\subsection{LISA}

The Primary objective of Laser Interferometer Space Antenna (LISA) mission is to detect and observe gravitational waves from massive black holes (MBHs) and galactic binaries such as pair of close white dwarfs, pair of neutron stars in the frequency range of $10^{-4}$ to $10^{-1} \mathrm{~Hz}$. This low frequency gravitational wave is inaccessible to ground base interferometer because of the local gravitational noise and limited base length of interferometer. Not only this LISA can also provide unique information about formation, growth, space density and the surroundings of massive black holes (MBHs).

LISA was initially a joint project of ESA/NASA to develop a space based gravitational wave detector [43]. 
NASA described the project as "one of the two large space mission" to be implemented in this decade. However in April 2011 NASA withdrew its partnership with the ESA due to funding limitation. The ESA (European Space Agency) had then planned to begin a full revision of mission concept in February 2012.

LISA mission can be described as a big Michelson interferometer, is comprised of three identical space crafts located $5 \times 10^{6} \mathrm{Km}$ apart forming an equilateral triangle. The center of triangle formation is IAU from the Sun and $20^{\circ}$ behind the Earth and inclined at $60^{\circ}$ to the ecliptic (as shown in Figure 6). The three LISA space crafts are to be launched on a single Delta-II 7925H. The initial orbit has an excess energy of $C_{3}=1.1 \mathrm{Km}^{2} / \mathrm{s}^{2}$ so that the three spacecraft's will drift slowly behind the Earth. The desired final orbits have semi major axis $a=1$ AU (150 $\times$ $10^{6} \mathrm{Km}$ ), separationd $=5 \times 10^{6} \mathrm{Km}$ eccentricity $e=\frac{2 d}{a^{3}}$ \& inclination $i= \pm \frac{2 d}{a}$. After reaching the final orbit the spacecraft will evolve under gravitational forces only.

LISA can be described as a big Michelson Interferometer, whose implementation in space is totally different from Laser Interferometer on the ground. LISA will be sensitive enough to detect gravitational waves induced strain of amplitude $h=\frac{\Delta l}{l}<10^{-23}$ in one year of observation with signal to noise ratio of 5 . The LISA relies on unusual flight technologies like drag free flight and precision measurement technique to shield the proof masses from the adverse effects due to the solar radiation pressure. Nevertheless sensitivity of LISA is determined by a wide range of noise sources like optical path noise due to fake's fluctuations in the lengths of the optical paths and acceleration noise which is due to forces acting on the proof masses.

Figure 7 show typical sensitivity curve wherein it is observed that in the low frequency range say $2 \mathrm{mHz}$, the noise and thus the sensitivity is determined by the acceleration noise, leading to a decrease in sensitivity towards lower frequencies roughly proportional to $\mathrm{f}^{-2}$. Above about $2 \mathrm{mHz}$, the noise is dominated by the shot noise where by the decline of the antenna transfer function above $10 \mathrm{mHz}$ causes a decrease in sensitivity roughly proportional to the frequency.

There are many other issues associated with LISA which can be referred to [44] [45] by the readers.

The baseline mission design for LISA was finalized in 2005. According to present status LISA is in the Cosmic Vision 2015-2025 programme and likely shall be launched in 2020. The key technologies for LISA are being testing on the LISA path finder mission [46]. LISA path finder will fly the LISA Technology Package (LTP) which is consisting of LISA arm of $38 \mathrm{~cm}$. The three main tasks of LISA path finder are to measure the acceleration phase noise, readout noise and noise caused by the departure of the Doppler link from the ideal scheme. It is designed to test the accuracy of these to within an order of magnitude of that required by the full LISA. Path finder is scheduled for launch in mid-2014 after which it will orbit the $L_{1}$ point with a 180 day mission plan.

\section{Future Prospectus}

Detection of gravitational waves is a startling paradox. As on date no one has actually detected a gravitational

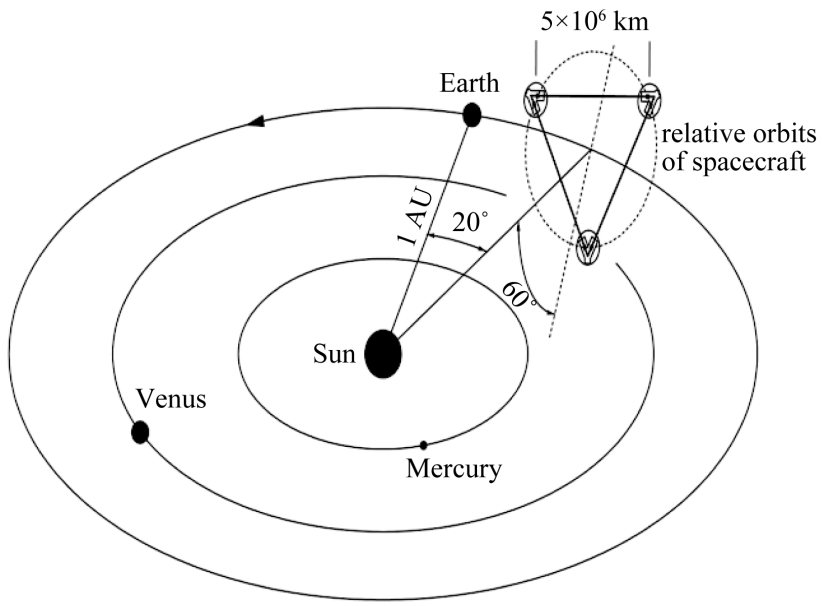

Figure 6. The proposed LISA detector. Courtesy: www.lisa.nasa.gov. 


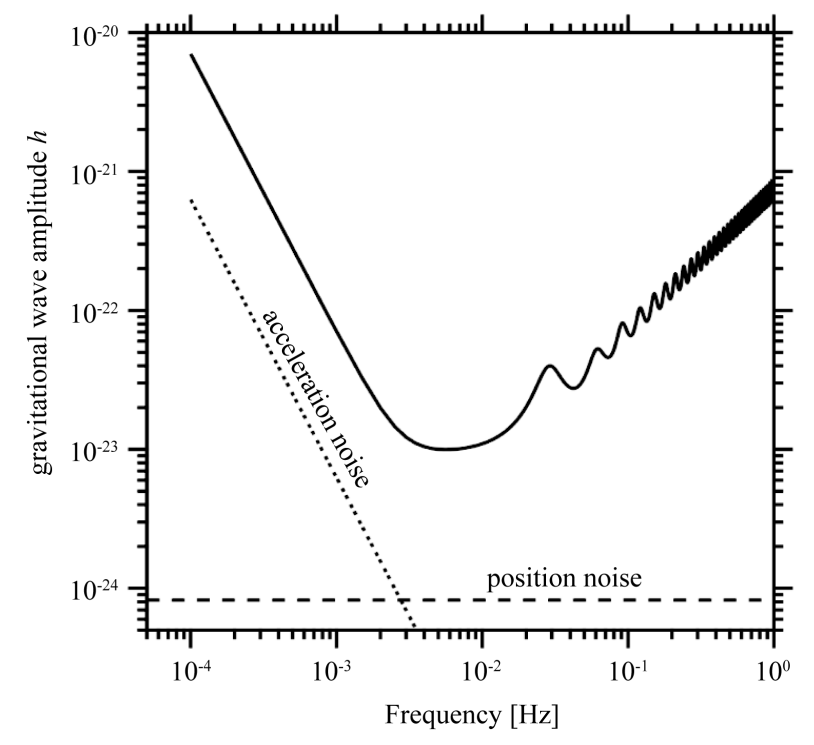

Figure 7. LISA sensitivity for one year integration time and a signal-to noise ratio of 5 , averaged over all possible source locations and polarizations. Also indicated are the position noise (dashed line) and the acceleration noise (dotted line). Courtesy: www.lisa.nasa.gov.

wave. "Harald Luck" a Scientist at Geo600 explained; "Einstein in general theory of relativity predicted the existence of gravitational waves with a remark that we would never be able to observe them as they would be too weak to be detected by the time they reach Earth”. Scientific world intend to prove Einstein right in the first instance and wrong in the second.

For years scientist have been trying \& failing to detect gravitational waves, but even though scientists remained confident by exploiting the precision of laser interferometry.

It is well known fact that dark matter hypothesis is mainly based on the observation of its gravitational effects \& hence the gravitational windows seems to be the most promising discovery tool for future investigation of universe. It is possible by a new generation of interferometers. The time scale for up gradation of current detectors is roughly 30 years.

For the high frequency region of the spectrum (from approximately $1 \mathrm{~Hz}$ to $10 \mathrm{kHz}$ ), we foresee the evolution and expansion of the current LIGO-VIGRO-GEO network into a worldwide alliance of interferometric detectors operating in co-ordination to extract the maximal scientific returns. The second generation detectors like Advanced LIGO (aLIGO), Advanced VIRGO \& GEO-HF) form a solid basis for the detections and surveys of gravitational waves sources in the $10 \mathrm{~Hz}-10 \mathrm{kHz}$ regime [47]-[49]. In fact by 2015, several parts of the detectors will be replaced to improve the sensitivity by a factor of ten. New lasers with large power, new mirrors (heavier, more reflective and with a high level of perfection), new optical and mechanical components will be installed to achieve this difficult target. An improvement by a factor of 10 - 15 times in sensitivity corresponds to an increase by a factor of thousand in detection rate in one year of operation of the advanced detectors at the nominal sensitivity, about 40 coalescences of neutron star binary systems are expected to be detected [50] [51]. The advance detectors capability to detect a coalescence of a binary neutron star system at a distance of about 140 MPC and a coalescence of binary systems of black holes at a distance of about 1 GPC will open up a gravitational wave astrophysics era. Advanced LIGO will apply some of the technologies from the GEO600 interferometer, such as the use of a signal recycling mirror at the output port and monolithic silica suspensions for the test masses, instead of the current steel wire slings. Larger test masses will be used with an increase from 11 to $40 \mathrm{~kg}$, although the masses will still be made from fused silica.

To overcome photon shot noise, due to the quantum nature of light, squeezed states have been injected to improve the performance of the LIGO beyond the quantum noise limit, most notably in the frequency region down to $150 \mathrm{~Hz}$. With the injection of squeezed states, this LIGO detector shall demonstrate the best broadband sensitivity to gravitational waves ever achieved, with important implications for observing the gravitational-wave 
Universe with unprecedented sensitivity [52].

The mirror coating is likely to consist of multiple alternating layers of silica and tantala, with the tantala layers doped with titania to reduce the coating thermal noise. The seismic isolation systems will be replaced with improved versions offering a seismic cut-off frequency of $\sim 10 \mathrm{~Hz}$ as opposed to the current cut-off of $\sim 40 \mathrm{~Hz}$. Advance Virgo will apply similar upgrades to those for a LIGO and over a similar timescale. Plans are to add a signal recycling mirror, monolithic suspensions, increased laser power to $\sim 200 \mathrm{~W}$, improved coatings, and to potentially use non-Gaussian beams.

The immediate priority is to successfully build commission and operate the already planned second generation detectors. The approved second generation detectors seek higher sensitivity in the $100 \mathrm{~Hz}$ regime and extension to lower frequencies as the most promising and feasible directions to peruse. Moreover, the high priority for ground based gravitational wave detectors development is expansion of the network to add additional detectors with appropriately chosen intercontinental baselines and orientations to maximize the ability to extract source information. The question of how many detectors a network needs is best framed in the context of the value of additional detectors, as noted a network consisting only of LIGO-VIRGO-GEO has poor angular resolution along directions perpendicular to the line connecting the US and Europe and corresponding poor polarization information. To augment the second generation network, the most advanced plans for additional detectors centre on the Japanese large scale cryogenic gravitational wave telescope (LCGT) [53]-[55] and the Australian international Gravitational observatory (AIGO). Preliminary discussion has also begun as to the possibility of a detector located in India (INDIGO).

LCGT is a proposed $3 \mathrm{~km}$ detector. The interferometeric optical configuration and planned laser power is similar to that of Advanced LIGO and Advanced VIRGO giving LCGT comparable sensitivity in the high frequency region. However LCGT would consist of two interferometer in one vacuum envelope. It has two exceptional features, one, it is located underground in the Kamiska mine about $220 \mathrm{~km}$ from Tokyo and second, it is planned to use cryogenic cooling for the interferometer mirrors. Cryogenic cooling of the interferometer optics offers many benefits (reduce thermal noise, reduce thermal lensing in the subtracts). The underground location offers a significantly lower seismic noise level and should greatly improve the low frequency performance.

The LCGT design is at an advanced stage. Considerable enabling research and development (R\&D) has been completed and an experienced team of gravitational wave experiments have developed the concept. Funding for the first phase of construction of LCGT was announced in June 2010 [56].

AIGO, Australian international gravitational observatory is a surface facility; the current favored location is about $80 \mathrm{kms}$ from Perth in Western Australia. The site can accommodate a $5 \mathrm{~km}$ detector, although the current design has $4 \mathrm{~km}$ arm. The planned configuration is similar to Advance LIGO and Advance VIRGO. The leading scientific organization for AIGO, ACIGA, is a member of the LIGO scientific collaboration and thus is familiar with the advanced LIGO design.

Based on the argument outlined above for the desired number and location of additional second generation network detectors, LCGT and AIGO would constitute excellent addition to the global gravitational wave network. The design strain amplitude sensitivity curve for a LIGO, Advance Virgo and LCGT is shown in Figure 8.

Successful deployment of third generation underground gravitational wave observatories will require development of a number of new technologies by the Gravitational wave community. Many of the necessary research and development programmes are taken in a limited number of places, like Europe and USA. Thanks to the support of the European Commission, through a design study tool in the Seventh Frame work programme (FP7), the conceptual design of a 3rd generation Gravitational wave observatory has been realized, able to compete and collaborate with the most sensitivity optical telescopes. The Einstein gravitational wave telescope (ET) [57] began in 2008 to assess the feasibility of third generation detectors with a largely improved sensitivity especially below $10 \mathrm{~Hz}$ due to an underground location. This new infrastructure aimed to be operative in the 2020 decade, will test the cosmological model of the universe using gravitational wave signals, thanks to its capability to see many source at large red shift [58]. ET will be a wonderful proofing tool of the general relativity prediction in all radioactive process involving intense gravitational fields, like in the presence of intermediate mass black holes. It will allow detailed investigation of the nature of isolated neutron star looking both to the continuous emission of the Pulsars and to the explosion of Supernovae. Investigations into the interferometric configuration have already been studied [59], with suggestions including a triple interferometer system made up from an equilateral triangle, an underground location, and potentially a xylophone configuration. Three potential sensitivity 


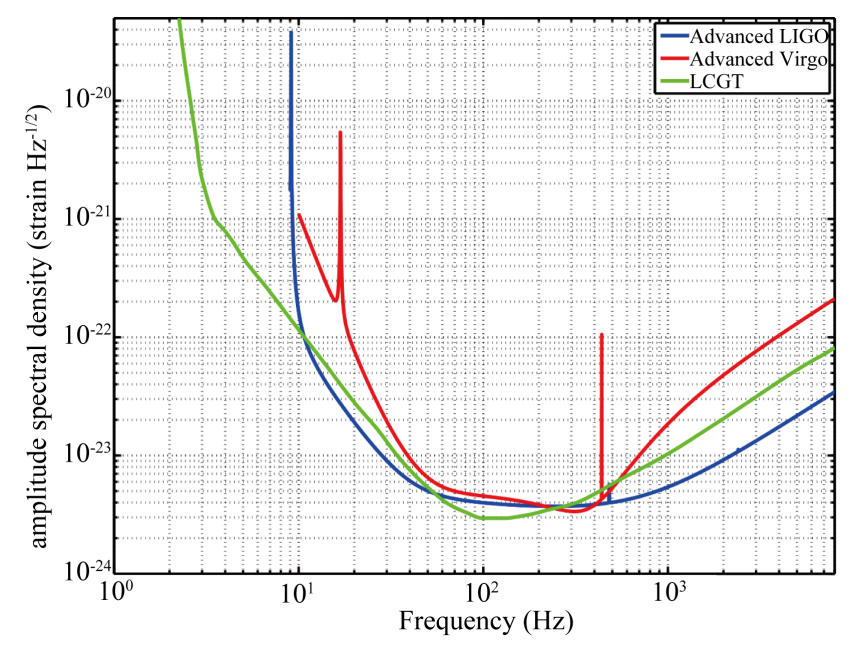

Figure 8. Design sensitivity curves for the Advanced LIGO, Advanced Virgo and LCGT second generation detectors. Courtesy: www.ligo.caltech.edu.

curves are plotted for different configurations of detectors (as shown in Figure 9).

Inconveniently, due to seismic noise and baselines length issue, the low frequency $<10 \mathrm{~Hz}$ part of the spectrum, where the most interesting events are expected will not be accessible. The space based detector LISA was conceived to fill the gap extending the observational capability to about $10^{-4} \mathrm{~Hz}$. Due to mission cost growth and severe budget constraints, a flight prior to 2020 now seems very unlikely.

Another space-born interferometer, the DECI-Hertz Interferometer gravitational waves observatory (DECIGO) has been proposed by Seto, Kawamara \& Nakamura [60]. It is gravitational wave antenna in space in the 0.1 to $10 \mathrm{~Hz}$ frequency band. DECIGO [61] [62] is a Japanese project designed to fill the gap in frequency between ground-based detectors and LISA. Its objective is to directly observe the beginning of the universe, thus possibly reveal its mysterious origin through detection of gravitational wave. It consists of three drag free spacecraft's $1000 \mathrm{Km}$ apart from each other. It is due for launch in 2027. Although still early in its design there are plans for two precursor technology demonstration missions (DECIGO Pathfinder [63] and Pre-DECIGO), with a main mission having a launch date in the mid-2020s. A similar mission suggested is the US Big Bang Observer (BBO) [64] [65]. Its main aims will be to detect the stochastic background from the early universe, but it can also be used for high precision cosmology [66]. The current configuration would consist of three LISA-like constellations of three spacecraft each, with $50000 \mathrm{~km}$ arm lengths, and separated in their orbit by $120^{\circ}$. The launch of this mission would be after DECIGO.ASTROD-GW (Astrodynamical space test of Relativity using optical devices optimized of GW detection) is another space based gravitational wave interferometric mission [67]. It consists of three satellites that are placed at near Lagrange points L3, L4, \& L5 respectively farming a large triangular interferometer similar to LISA. Its arm length is 52 times larger than that of LISA therefore it is more sensitive than LISA at lower frequency.

Further, there is another Chinese future space mission called ASTROD in frequency range $\sim 0.1 \mu \mathrm{Hz}$ to $1 \mathrm{mHz}$, which is expected to be launched in 2021 [68].

However a 30 years programme in low frequency gravitational wave astronomy should set as its goal for the development and implementation of three generations of space antennas one per decade.

For the first decade observatory, we make the case for a scaled down mission of the LISA-2020, that is comparable to cost and duration to medium scale astrophysics missions such as the 1978 (\$630M) Einstein (HEAO2) X-ray observatory [69], the 1989 (\$680M) COBE cosmic background Explorer [70] and the 1999 fuse for ultraviolet spectroscopic explorer. We find a mission of this class is possible if the measurement requirements are somewhat relaxed and baseline smaller than LISA is used. Such a mission could be launched by 2020 using a conventional program development plane, possibly including international collaborations.

A second decade observatory could be either an enhanced LISA type technology observatory, the most ambitious and costly eLISA or an upgraded version thereof or an instrument based on alternative technologies, if available on a timely basis. 


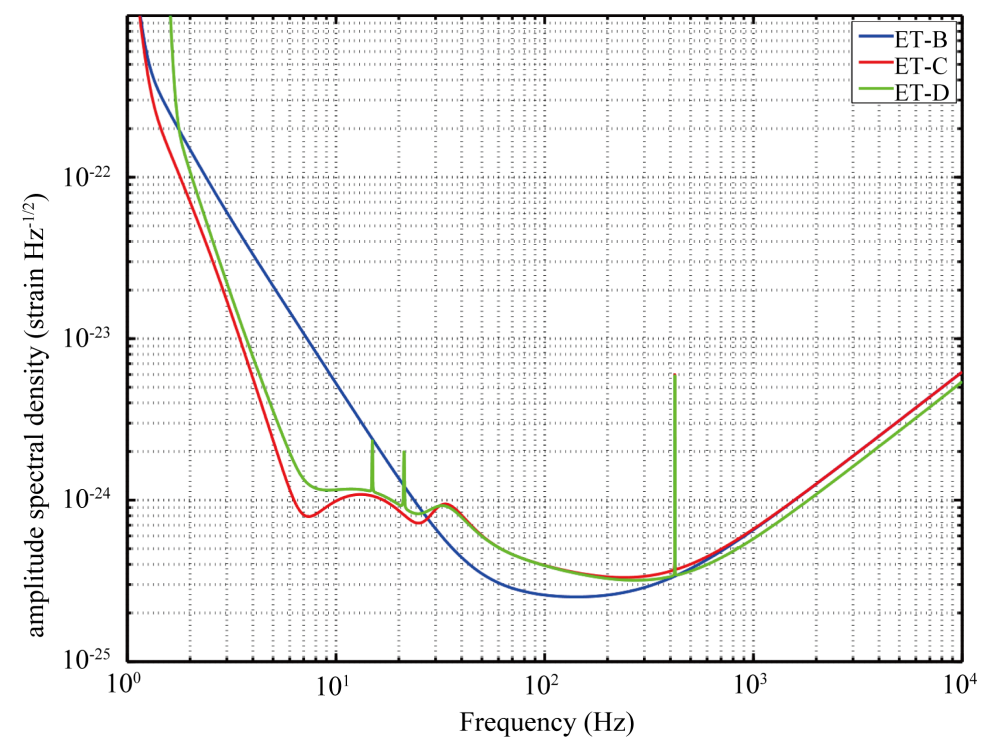

Figure 9. Potential sensitivities of the Einstein Telescope for 3 different design concepts: ET-B, ET-C and ET-D. Courtesy: www.lisa.nasa.gov.

For third decade observatory one would expect new technological developments for second and third generation low frequency antennas that would proceed in parallel with the implementation of the previous instruments.

\section{Conclusions}

The field of gravitational wave science is on the verge of direct observation of the waves predicted by Albert Einstein in general theory of relativity. In the coming decades, ultra sensitive arrangement of ground based interferometers and complementary space based interferometers observing the gravitational wave sky will discover entirely unexpected phenomena which will provide new insight into many of the most profound astrophysical phenomena known. This new gravitational window into the cosmos would radically change the human approach towards the understanding of universe.

The ground based gravitational wave detectors currently taking data and the advanced detectors that will be brought on line over the next three to seven years, will be critical in establishing the field of gravitational astronomy through the detection of gravitational waves. The first priority for ground based gravitational wave detector development is expansion of the network as begun by LIGO, VIRGO and GEO and their planned upgrades. In broader terms, there is an important opportunity to further expand the scientific reach of this ground based network by developing underground interferometers with even better sensitivity and frequency coverage.

Many gravitational wave sources only radiate at much lower frequencies. The most promising route to access these is to fly a laser interferometers in space. The scientific reach of such a space based instruments would be complementary to the ground based array. Given the time scale in which gravitational wave astronomy will produce results, we now have an excellent opportunity for our field to foster the interest of the current and coming generation of astronomers and astrophysics in the scientific potential of our field.

Finally, we note that gravitational wave research has always been a technology intensive field, driving technology in some area, while benefitting from developments in others. The need to stretch the limits of sensitivity has resulted in developments that have profited other field of science and technology, notably precision measurement, metrology, optics, laser space technology etc. There is no doubt that this highly productive interchange between gravitational wave science and other spheres of science and technology will continue providing tangible benefits to society, in addition to the intellectual satisfaction of an improved knowledge of our universe.

\section{Acknowledgements}

One of us (MONGA SHOWKAT) put on record that department of Higher Education has allowed him to proceed on leave for a period of 4 months for this work. Thanks to the concerned department for this generosity. 


\section{References}

[1] Taylor, J.H., Fowler, L.A. and McCulloch, P.M. (1979) Measurements of General Relativistic Effects in the Binary Pulsar PSR 1913+16. Nature, 277, 437-440. http://dx.doi.org/10.1038/277437a0

[2] Taylor, J.H. (1994) Binary Pulsar and Relativistic Gravity. Review of Modern Physics, 66, 711719. http://dx.doi.org/10.1103/RevModPhys.66.711

[3] Hulse, R.A. and Taylor, J.H. (1974) A High Sensitivity Pulsar Survey. The Astrophysical Journal, 191, 59-61. http://dx.doi.org/10.1086/181548

[4] Hulse, R.A. and Taylor, J.H. (1975) Discovery of a Pulsar in a Binary System. The Astrophysical Journal, 195, 51-53. http://dx.doi.org/10.1086/181708

[5] Hulse, R.A. and Taylor, J.H. (1975) A Deep Sample of Pulsar and Their Spatial Extent in the Galaxy. The Astrophysical Journal, 201, 55-59. http://dx.doi.org/10.1086/181941

[6] Damour, T. and Esposito, F.G. (1996) Tensor-Scalar Gravity and Binary-Pulsar Experiments. Physical Review D, 54, 1474-1491. http://dx.doi.org/10.1103/PhysRevD.54.1474

[7] Damour, T. and Esposito, F.G. (1993) Nonperturbative Strong-Field Effects in Tensor-Scalar Theories of Gravitation. Physical Review Letters, 70, 2220-2223. http://dx.doi.org/10.1103/PhysRevLett.70.2220

[8] Will, C.M. (1977) Gravitational Radiation from Binary Systems in Alternative Metric Theories of Gravity—Dipole Radiation and the Binary Pulsar. Astrophysical Journal, 214, 826-839. http://dx.doi.org/10.1086/155313

[9] Krauss, L.M., Dodelson, S. and Meyer, S. (2010) Primordial Gravitational Waves and Cosmology. Science, 328, 989992. http://dx.doi.org/10.1126/science.1179541

[10] Weber, J. (1960) Detection and Generation of Gravitational Waves. Physical Review D, 117, 306-313. http://dx.doi.org/10.1103/PhysRev.117.306

[11] Gertsenshtein, M.E. and Pustovoit, V.I. (1963) On the Detection of Low-Frequency Gravitational Waves. Soviet Physics-JETP, 16, 433-435.

[12] Abbott, B.P., Abbott, R., Acernese, R., Ajith, P., Allen, B., Alshourbagy, M., Amin, R.S., Anderson, S.B., et al. (2010) Searches for Gravitational Waves from Known Pulsars with Science Run 5 LIGO Data. The Astrophysical Journal, 713, 671-685. http://dx.doi.org/10.1088/0004-637X/713/1/671

[13] Ni, W.T. (2008) Astrod and Astrod i-Overview and Progress. International Journal of Modern Physics, 17, $921-940$. http://dx.doi.org/10.1142/S0218271808012619

[14] Ni, W.T. (2005) Empirical Foundations of the Relativistic Gravity. International Journal of Modern Physics D, 14, 901-921. http://dx.doi.org/10.1142/S0218271805007139

[15] Farmer, A.J. and Phinney, E.S. (2003) The Gravitational Wave Background from Cosmological Compact Binaries. Monthly Notices of the Royal Astronomical Society, 346, 1197-1214. http://dx.doi.org/10.1111/j.1365-2966.2003.07176.x

[16] Armstrong, J.W., Estabrook, F.B. and Tinto, M. (1999) Time-Delay Interferometry for Space-Based Gravitational Wave Searches. Astrophysical Journal, 527, 814-826. http://dx.doi.org/10.1086/308110

[17] Cutler, C. (1998) Angular Resolution of the LISA Gravitational Wave Detector. Physical Review D, 57, $7089-7102$. http://dx.doi.org/10.1103/PhysRevD.57.7089

[18] Kawamura, S., Nakamura, T., Ando, M., Seto, N., Tsubono, N., Numata, K., Takahashi, R., Nagan, S., Ishikawa, T., Musha, M., Ueda, K., et al. (2006) The Japanese Space Gravitational Wave Antenna-DECIGO. Classical and Quantum Gravity, 23, 125-131. http://dx.doi.org/10.1088/0264-9381/23/8/S17

[19] Ni, W.T. (2005) Empirical Foundations of the Relativistic Gravity. International Journal of Modern Physics, 14, 901921. http://dx.doi.org/10.1142/S0218271805007139

[20] Ni, W.T., Hanns, S., Laemmerzahl, C., Mingyong, H., Chengming, L. and He, P.J. (2010) ASTROD Optimized for Gravitational Wave Detection: ASTROD-GW. 38th COSPAR Scientific Assembly, Bremen, 18-15 July 2010, p. 7.

[21] Aasi, J., Abadie, J., Abbott, B.P., Abbott, R., Abbott, T., et al. (2013) Directed Search for Continuous Gravitational Waves from the Galactic Center. Physical Review D, 88, 102002. http://dx.doi.org/10.1103/PhysRevD.88.102002

[22] Belczynski, K., Kalogera, V. and Bulik, T. (2002) A Comprehensive Study of Binary Compact Objects as Gravitational Wave Sources: Evolutionary Channels, Rates, and Physical Properties. Astrophysical Journal, 572, 407-431. http://dx.doi.org/10.1086/340304

[23] Abbott, B., Abbott, R., Adhikari, R., Ageev, A., Allen, B., Amin, R., Anderson, S.B., Anderson, W.G., Araya, M., Armandula, H., et al. (2004) Detector Description and Performance for the First Coincidence Observations between LIGO and GEO. Nuclear Instruments and Methods in Physics Research Section A, 517, 154-179.

http://dx.doi.org/10.1016/j.nima.2003.11.124 
[24] Abbott, B., Abbott, R., Adhikari, R., Agresti, J., Ajith, P., Allen, B., Amin, R., Anderson, S.B., Anderson, W.G., Arain, M., et al. (2008) Search for Gravitational Waves from Binary Inspirals in S3 and S4 LIGO Data. Physical Review D, 77, 062002. http://dx.doi.org/10.1103/PhysRevD.77.062002

[25] Abbott, B., Abbott, R., Adhikari, R., Agresti, J., Ajith, P., Allen, B., Amin, R., Anderson, S.B., Anderson, W.G., Rain, M., Araya, M., et al. (2007) Search for Gravitational-Wave Bursts in LIGO Data from the Fourth Science Run. Classical and Quantum Gravity, 24, 5343-5369. http://dx.doi.org/10.1088/0264-9381/24/22/002

[26] Abbott, B., Abbott, R., Abbott, R., Gresti, J., Ajith, P., Allen, B., Amin, R., Anderson, S.B., Anderson, W.G., Arain, M., et al. (2007) Upper Limits on Gravitational Wave Emission from 78 Radio Pulsars. Physical Review D, 76, 042001. http://dx.doi.org/10.1103/PhysRevD.76.042001

[27] Abbott, B., Abbott, R., Adhikari, R., Agresti, J., Ajith, P., Allen, B., Amin, R., Anderson, S.B., Anderson, W.G., Araya, M., Armandula, H., et al. (2007) Searching for Stochastic Background of Gravitational Waves with LIGO. The Astrophysical Journal, 659, 918. http://dx.doi.org/10.1086/511329

[28] Abbott, B., Abbott, R., Adhikari, R., Ajith, P., Allen, B., Allen, G., Amin, R., Anderson, S.B., Anderson, W.G., Arain, M.A., Araya, M., et al. (2008) First Joint Search for Gravitational-Wave Bursts in LIGO and GEO 600 Data. Classical and Quantum Gravity, 25, 245008. http://dx.doi.org/10.1088/0264-9381/25/24/245008

[29] Acernese, F., Amico, P., Al-Shourbagy, M., Aoudia, S., Avino, S., Babusci, D., Ballardin, G., Barillé, R., Barone, F., Barsotti, L., Barsuglia, M., et al. (2005) Status of Virgo. Classical and Quantum Gravity, 22, 869-880. http://dx.doi.org/10.1088/0264-9381/22/18/S01

[30] Acernese, F., Amico, P., Alshourbagy, M., Antonucci, F., Aoudia, S., Avino, S., Babusci, D., Ballardin, G., Barone, F., Barsotti, L., Barsuglia, M., et al. (2006) The Status of Virgo. Classical and Quantum Gravity, 23, 635-690. http://dx.doi.org/10.1088/0264-9381/23/19/S01

[31] Acernese, F., Amico, P., Alshourbagy, M., Antonucci, F., Aoudia, S., Astone, P., Avino, S., Babusci, D., Ballardin, G., Barone, F., Barsotti, L., et al. (2007) Status of Virgo Detector. Classical and Quantum Gravity, 24, 381-388. http://dx.doi.org/10.1088/0264-9381/24/19/S01

[32] Abramovici, A., Althouse, W.E., Drever, R.W.P., Gursel, Y., Kawamura, S., Raab, F.J., Shoemaker, D., Sievers, L., spero, R.E., Thorne, K.S., et al. (1992) LIGO-The Laser Interferometer Gravitational-Wave Observatory. Science, 256, 325-333. http://dx.doi.org/10.1126/science.256.5055.325

[33] Bradaschia, C., del Fabbro, R., di Virgilio, A., Giazotto, A., Kautzky, H., Montelatici, V., Passuello, D., Brillet, A., Cregut, O., Hello, P., Man, C.N., et al.(1990) The VIRGO Project: A Wide Band Antenna for Gravitational Wave Detection. Nuclear Instruments and Methods in Physics Research Section A, 289, 518-525. http://dx.doi.org/10.1016/0168-9002(90)91525-G

[34] Abadie, J., Abbott, B.P., Abbott, R., Adhikari, R., Ajith, P., Allen, B., Allen, G., Amador, C.E., Amin, R.S., Anderson, S., et al. (2011) Beating the Spin-Down Limit on Gravitational Wave Emission from the Vela Pulsar. The Astrophysical Journal, 737, 93-108. http://dx.doi.org/10.1088/0004-637X/737/2/93

[35] Ando, M., Arai, K., Takahashi, R., Heinzel, G., Kawamura, S., Tatsumi, D., Kanda, N., Tagoshi, H., Araya, A., Barton, M.A., et al. (2001) Stable Operation of a 300-m Laser Interferometer with Sufficient Sensitivity to Detect Gravitational-Wave Events within Our Galaxy. Physical Review Letters, 86, 3950-3954. http://dx.doi.org/10.1103/PhysRevLett.86.3950

[36] Willke, B., Aufmuth, P., Aulbert, C., Babak, S., Balasubramanian, R., Barr, B.W., Berukoff, S., Bose, S., Cagnoli, G., et al. (2002) The GEO 600 Gravitational Wave Detector. Classical and Quantum Gravity, 19, 1377-1387. http://dx.doi.org/10.1088/0264-9381/19/7/321

[37] Smith, J.R., Allen, B., Aufmuth, P., Aulbert, C., Babak, S., Balasubramanian, R., Barr, B.W., Berukoff, S., Bunkowski, A., Cagnoli, G., et al. (2004) Commissioning, Characterization and Operation of the Dual-Recycled GEO 600. Classical and Quantum Gravity, 21, S1737-S1745. http://dx.doi.org/10.1088/0264-9381/21/20/016

[38] Willke, B., Aufmuth, P., Aulbert, C., Babak, S., Balasubramanian, R., Barr, B.W., Berukoff, S., Cagnoli, G., Cantley, C.A., Casey, M.M., et al. (2004) Status of GEO 600. Classical and Quantum Gravity, 21, S417-S423. http://dx.doi.org/10.1088/0264-9381/21/5/006

[39] Willke, B., Ajith, P., Allen, B., Aufmuth, P., Aulbert, C., Babak, S., Balasubramanian, R., Barr, B.W., Berukoff, S., Bunkowski, A., Cagnoli, G., et al. (2006) The GEO-HF Project. Classical and Quantum Gravity, 23, S207-S214. http://dx.doi.org/10.1088/0264-9381/23/8/S26

[40] Hild, S., Grote, H., Degallaix, J., Chelkowski, S., Danzmann, K., Freise, A., Hewitson, M., Hough, J., Luck, H., Prijatelj, M., Strain, K.A., Smith, J.R. and Willke, B. (2009) DC-Readout of a Signal-Recycled Gravitational Wave Detector. Classical and Quantum Gravity, 26, Article ID: 055012. http://dx.doi.org/10.1088/0264-9381/26/5/055012

[41] Yamamoto, K., Uchiyama, T., Miyoki, S., Ohashi, M., Kuroda, K., Ishitsuka, H., Akutsu, T., Telada, S., Tomaru, T., Suzuki, T., Sato, N., et al. (2008) Current Status of the CLIO Project. Journal of Physics: Conference Series, 122, Ar- 
ticle ID: 012002. http://dx.doi.org/10.1088/1742-6596/122/1/012002

[42] Akutsu, T., Ando, M., Haruyama, T., Kanda, N., Kuroda, K., Miyoki, S., Ohashi, M., Saito, Y., Sato, N., Shintomi, T., Suzuki, T., et al. (2008) Search for Continuous Gravitational Waves from PSR J0835-4510 Using CLIO Data. Classical and Quantum Gravity, 25, Article ID: 184013. http://dx.doi.org/10.1088/0264-9381/25/18/184013

[43] Danzmann, K. and the LISA Study Team (1996) LISA: Laser Interferometer Space Antenna for Gravitational Wave Measurements. Classical and Quantum Gravity, 13, 247-250. http://dx.doi.org/10.1088/0264-9381/13/11A/033

[44] Jennrich, O. (2009) LISA Technology and Instrumentation. Classical and Quantum Gravity, 26, Article ID: 153001. http://dx.doi.org/10.1088/0264-9381/26/15/153001

[45] Johann, U.A., Ayre, M., Gath, P.F., Holota, W., Marenaci, P., Schulte, H.R., Weimer, P. and Weise, D. (2008) The European Space Agency's LISA Mission Study: Status and Present Results. Journal of Physics: Conference Series, 122, Article ID: 012005.http://dx.doi.org/10.1088/1742-6596/122/1/012005

[46] Armano, M., Benedetti, M., Bogenstahl, J., Bortoluzzi, D., Bosetti, P., Brandt, N., Cavalleri, A., Ciani, G., Cristofolini, I., Cruise, A.M., et al. (2009) LISA Pathfinder: The Experiment and the Route to LISA. Classical and Quantum Gravity, 26, Article ID: 094001.

[47] Blair, D.G., Howell, E.J., Ju, L. and Zhao, C. (2012) Advanced Gravitational Wave Detectors. Cambridge University Press, Cambridge.

[48] Acernese, F., Amico, P., Arnaud, N., Arnault, C., Babusci, D., Ballardin, G., Barone, F., Barsuglia, M., Bellachia, F., Beney, J.L., et al. (2002) The Present Status of the VIRGO Central Interferometer. Classical and Quantum Gravity, 19, 1421-1428. http://dx.doi.org/10.1088/0264-9381/19/7/325

[49] Harry, G.M. (2010) Advanced LIGO: The Next Generation of Gravitational Wave Detectors. Classical and Quantum Gravity, 27, Article ID: 084006. http://dx.doi.org/10.1088/0264-9381/27/8/084006

[50] Abadie, J., Abbott, B.P., Abbott, R., Abernathy, M., Accadia, T., Acernese, F., Adam, C., Adhikari, R., Ajith, P., Allen, B., et al. (2010) Predictions for the Rates of Compact Binary Coalescences Observable by Ground Based Gravitational Wave Detectors. Classical and Quantum Gravity, 27, Article ID: 173001. http://dx.doi.org/10.1088/0264-9381/27/17/173001

[51] Kopparapu, R.K., Hanna, C., Kalogera, V., Shaughnessy, R.O., Gonzalez, G., Brady, P.R. and Fairhurst, S. (2008) Host Galaxies Catalog Used in LIGO Searches for Compact Binary Coalescence Events. Astrophysical Journal, 675, 1459-1467. http://dx.doi.org/10.1086/527348

[52] Aasi, J., Abadie, J., Abbott, B.P., Abbott, R., Abbott, T.D., Bernathy, M.R., Adam, C., Adam, T., Addesso, P. and Adhikar, R.X. (2013) Enhanced Sensitivity of the LIGO Gravitational Wave Detector by Using Squeezed States of Light. Nature Photonics, 7, 613-619. http://dx.doi.org/10.1038/nphoton.2013.177

[53] Miyoki, S. (2005) Large Scale Cryogenic Gravitational Wave Telescope. Nuclear Physics B, 138, 439-442. http://dx.doi.org/10.1016/j.nuclphysbps.2004.11.101

[54] Ohashi, M. (2008) Status of LCGT and CLIO. Journal of Physics: Conference Series, 120, Article ID: 032008. http://dx.doi.org/10.1088/1742-6596/120/3/032008

[55] Kuroda, K. (2010) Status of LCGT. Classical and Quantum Gravity, 27, Article ID: 084004. http://dx.doi.org/10.1088/0264-9381/27/8/084004

[56] Uchiyama, T., Kuroda, K., Ohashi, M., Miyoki, S., Ishitsuka, H., Yamamoto, K., Hayakawa, H., Kasahara, K., Fujimoto, M.K., Kawamura, S., et al. (2004) Present Status of Large-Scale Cryogenic Gravitational Wave Telescope. Classical and Quantum Gravity, 21, 1161-1172. http://dx.doi.org/10.1088/0264-9381/21/5/115

[57] Gair, J.R., Mandel, I., Millerand, M. and Volonteri, M. (2011) Exploring Intermediate and Massive Black-Hole Binaries with the Einstein Telescope. General Relativity and Gravitation, 43, 485-518. http://dx.doi.org/10.1007/s10714-010-1104-3

[58] Amaro, S.P. and Santamaria, L. (2010) Detection of IMBHS with Ground-Based Gravitational Wave Observatories: A Biography of a Binary of Black Holes, from Birth to Death. Astrophysical Journal, 722, 1197-1206. http://dx.doi.org/10.1088/0004-637X/722/2/1197

[59] Freise, A., Chelkowski, S., Hild, S., DelPozzo, W., Perreca, A. and Vecchio, A. (2009) Triple Michelson Interferometer for a Third-Generation Gravitational Wave Detector. Classical and Quantum Gravity, 26, Article ID: 085012. http://dx.doi.org/10.1088/02649381/26/8/085012

[60] Allen, Z.A., Astone, P., Baggio, L., Busby, D., Bassan, M., Blair, D.G., Bonaldi, M., Bonifazi, P., Carelli, P. and Cerdonio, M. (2000) First Search for Gravitational Wave Bursts with a Network of Detectors. Physical Review Letters, 85, 50465050.http://dx.doi.org/10.1103/PhysRevLett.85.5046

[61] Sato, S., Kawamura, S., Ando, M., Nakamura, T., Tsubono, K., Araya, A., Funaki, I., Ioka, K., Kanda, N., Moriwaki, S., Musha, M., et al. (2009) DECIGO: The Japanese Space Gravitational Wave Antenna. Journal of Physics: Conference 
Series, 154, Article ID: 012040. http://dx.doi.org/10.1088/1742-6596/154/1/012040

[62] Kawamura, S., Ando, M., Seto, N., Sato, S., Nakamura, T., Tsubono, K., Kanda, N., Tanaka, T., Yokoyama, J., Funak, I., et al. (2011) The Japanese Space Gravitational Wave Antenna: DEECIGO. Classical and Quantum Gravity, 28, Article ID: 094011. http://dx.doi.org/10.1088/0264-9381/28/9/094011

[63] Ando, M., Kawamura, S., Sato, S., Nakamura, T., Tsubono, K., Araya, A., Funaki, I., Ioka, K., Kanda, N., Moriwaki, S., et al. (2009) DECIGO Pathfinder. Classical and Quantum Gravity, 26, Article ID: 094019. http://dx.doi.org/10.1088/0264-9381/26/9/094019

[64] Crowder, J. and Cornish, N.J. (2005) Beyond LISA: Exploring Future Gravitational Wave Missions. Physical Review D, 72, Article ID: 083005. http://dx.doi.org/10.1103/PhysRevD.72.083005

[65] Harry, G.M., Fritschel, P., Shaddock, D.A., Folkner, W. and Phinney, E.S. (2006) Laser Interferometry for the Big Bang Observer. Classical and Quantum Gravity, 23, 4887-4894. http://dx.doi.org/10.1086/157110

[66] Cutler, C. and Holz, D.E. (2009) Ultrahigh Precision Cosmology from Gravitational Waves. Physical Review D, 80, Article ID: 104009. http://dx.doi.org/10.1103/PhysRevD.80.104009

[67] Ni, W.T. (2013) Dark Energy, Co-Evolution of Massive Black Holes with Galaxies, and ASTROD-GW. Advances in Space Research, 51, 525-534. http://dx.doi.org/10.1016/j.asr.2012.09.019

[68] Braxmaier, C., Dittus, H., Foulon, B., Goklu, E., Grimani, C., Guo, J., Herrmann, S., Lammerzahl, C., Ni, W.T., Peter, A., et al. (2012) Astrodynamical Space Test of Relativity Using Optical Devices I (ASTROD I) -A Class-M Fundamental Physics Mission Proposal for Cosmic Vision 2015-2022. Experimental Astronomy, 34, 181-201. http://dx.doi.org/10.1007/s10686-011-9281-y

[69] Giacconi, R., Branduardi, G., Breil, U., Epstein, A., Fabricant, D., Feigelson, E., Forman, W., Gorenstein, P., Grindlay, J. and Gursky, H. (1979) The Ei/HEAO 2/ X-Ray Observatory. Astrophysical Journal, 230, 540-550. http://dx.doi.org/10.1086/157110

[70] Mather, J.C., Hauser, M.G., Benne, L., Boggess, W., Cheng Jr., E.S., Eplee, R.E., Freudenreich, H.T., Isaacman, B., et al. (1991) Early Results from the Background Explorer (COBE). Advance in Space Research, 11, 181-191. http://dx.doi.org/10.1016/0273-1177(91)90489-7 